\title{
Wavelet Transform-based Remote Sensing Image Compression
}

\author{
Mei-shan $\mathrm{Li}^{1}$, Yue Liü ${ }^{1 \star}$, Hong Zhang ${ }^{2}$ \\ ${ }^{1}$ College of Information Science \& Electronic Technology, Jiamusi University, \\ Jiamusi,154007,China \\ ${ }^{2}$ Graduate School, Jiamusi University, Jiamusi, 154007, China \\ e-mail: limeishanz@163.com, liuyue197612@126.com, zhanghongzc1@163.com \\ *Corresponding author
}

Keywords: Wavelet Transform;SPIHT;Remote Sensing Image Compression

\begin{abstract}
After analyzing characteristics of remote sensing images, the Fourier transform and wavelet transform are studied in this paper. It is found that the wavelet transform provides excellent properties and is more suited for image compression. Therefore, the wavelet transform is used to perform three-level decomposition on images. Next, SPIHT, an embedded encoding algorithm, is used to effectively compress remote sensing images. Extensive simulations show that this algorithm greatly outperform the traditional compression algorithms in terms of peak signal noise ratio (PSNR) and the quality of reconstructed images.
\end{abstract}

\section{Introduction}

The advancements of remote sensing techniques in recent years impose higher demands on compression effectiveness of remote sensing images. But the remote sensing images are characterized by high resolution, weak correlation, textural variety and complexity, high information quantity and code rate. Many compression algorithms have been proposed to achieve good compression ratio and distortion as well as meet data transmission and storage needs. These methods have pros and coins but none of them can compress as effectively as expected. In this context, wavelet transform comes into being.

After analyzing the characteristics of wavelet transform, this paper aims to fully tap the potential of wavelet transform, compressing several remote sensing images of resolution crowd and airport terminal via SPIHT.

\section{Wavelet Transform}

Wavelet transform is a novel time-frequency signal analysis method. Compared with Fourier analysis, wavelet transform provides good localization properties in both time and frequency domains. By applying increasingly finer time- or frequency-domain sampling step to the highfrequency components, it can focus on any detail of the image, and so it is known as the mathematical microscope. While processing images, wavelet transform can be used to decompose the image into sub-band signals with different spatial resolutions, frequency characters and directional properties. This enables low-frequency long-time features and high-frequency short-time features to be processed simultaneously, making decomposition of image signals more suited for human vision system characteristics and image data compression requirements.

Unlike the traditional encoding process of compression algorithms, the wavelet transform-based encoding process ${ }^{[1,2]}$ is given in Figure 1.

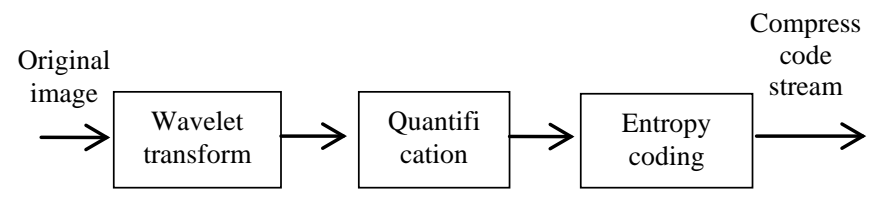

Fig.1 Wavelet transform-based encoding process 


\section{Compressed Encoding}

Currently, all traditional wavelet encoder falls into the transform coding type. It mainly consists of decorrelation transformation process, quantification process and entropy coding process. Many works are being done on wavelet encoders taking into account these three processes. The basic idea of wavelet encoding is to concentrate the energies of the original image on a few number of wavelet coefficients by performing 2D wavelet transformation on the original image. Therefore, the simplest approach to quantify coefficients is to abandon coefficients less than a threshold or define them as a constant. Because only wavelet coefficients that represent high energies are kept, data is compressed.

The most prominent method for quantifying wavelet coefficients is the embedded encoding method, including $\mathrm{EZW}^{[4]}$ (Embedded Zero-tree Wavelets), SPIHT ${ }^{[3,5]}$ (Set Partitioning In Hierarchical Trees), and EBCOT ${ }^{[6]}$ (Embedded Block Coding with Optimization Truncation). EZW and SPIHT are both based on spatial wavelet direction tree. These two algorithms are extensively simulated in this paper. Results demonstrate SPIHT is more suited to compress remote sensing images.

Said and Pearlman originated the well-known SPIHT algorithm in 1996. Hierarchical tree ${ }^{[7]}$ is the set of null trees that record positions of unimportant coefficients. The structure of null trees varies according to degrees of quantification errors. Set partitioning refers to the situation where the null trees change constantly, because there are important coefficients being continuously separated from the set of unimportant coefficients when the quantification errors decease. SPIHT provides a progressive coded transmission process where quantification errors decrease gradually, the set of unimportant coefficients is partitioned constantly and the null trees evolve constantly.

This algorithm can classify wavelet coefficients via set partitioning, introducing linked table to record the coefficients and sets acquired during previous search. The core of SPIHT is based on the assumption that if the coefficient of the low-frequency sub-band is subordinate to a threshold, then it is highly probable that the coefficient of the high-frequency sub-band is also subordinate to the threshold. Hence SPIHT is an improved version of EZW, it inherits the spatial orientation tree structure in Figure 2.

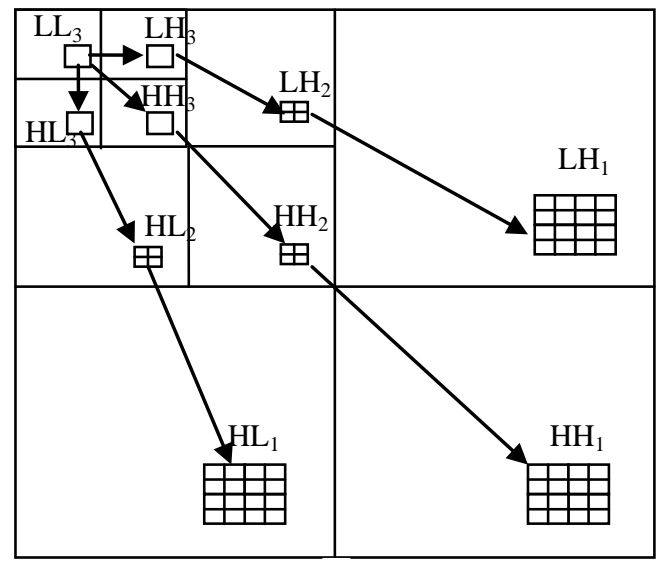

Fig.2 spatial orientation wavelet tree

Key elements of SPIHT are as follows:

1) The classification algorithm for partitioning sets of unimportant coefficients according to magnitudes of coefficients.

2) Gradual and orderly transmission of bit planes.

3) Construct zero tree using self-similarities among frequency bands.

SPIHT needs to define three linked tables: less important set linked table (LIS), important pixel linked table (LSP), less important pixel linked table (LIP). The algorithm undergoes two steps for each threshold: scanning process and refinement process. The algorithm uses $S_{n}(T)$ in the following function as a flag to evaluate the importance of the set 


$$
S_{n}(T)=\left\{\begin{array}{rr}
1, & \max _{(i, j) \in T}\left\{\left|c_{i, j}\right|\right\} \geq 2^{n} \\
0, & \text { otherwise }
\end{array}\right.
$$

Where $T$ denotes the set of coordinates. If $T$ is merely a pixel $(i, j)$, then $S_{n}(i, j)$ is used as a substitute for $S(\{(i, j)\})$.

\section{Experimental Result and Analysis}

In the experiment, several remote sensing images are used as the test images. The remote sensing images of resolution crowd are listed here, the size of which is $256 \times 256$ each. For test images, we begin with performing three-level wavelet decomposition with the $\mathrm{db} 4$ wavelet, and then carry out simulations using SPIHT and EZW. Experimental data is given in Table 1, data analysis is shown in Figure 3-5,and the reconstructed images are shown in Figure 6. Where PSNR ${ }^{[8]}$ is a measure of the image reconstruction quality, and it is also an important metrics for evaluating the performance of various image compression methods. The higher the PSNR value, the higher the image reconstruction quality. The PSNR is given by:

$$
P S N R=10 \lg \frac{255^{2}}{M S E}
$$

Its unit is $\mathrm{dB}$, and

$$
M S E=\frac{1}{M \times N} \sum_{i=0}^{M-1} \sum_{j=0}^{N-1}(x(i, j)-\hat{x}(x, j))^{2}
$$

$M$ and $N$ denote the length and width of the image in pixels, respectively. $x(i, j)$ denotes the value of $(i, j)$ in the original image, $\hat{x}(x, j)$ denotes the value of $(i, j)$ in the reconstructed image.

TABLE I. Comparison of experimental results

\begin{tabular}{|c|c|c|c|c|c|c|}
\hline \multirow{2}{*}{ bPp } & \multicolumn{4}{|c|}{ EZW } & \multicolumn{3}{c|}{ SPIHT } \\
\cline { 2 - 7 } & PSNR (db) & Encoding time (s) & Decoding time (s) & PSNR (db) & Encoding time (s) & Decoding time (s) \\
\hline 0.21 & 26.35 & 8.97 & 7.64 & 33.41 & 1.90 & 0.47 \\
\hline 0.32 & 28.37 & 14.58 & 8.07 & 34.45 & 3.25 & 1.06 \\
\hline 0.42 & 29.56 & 28.56 & 14.32 & 35.36 & 5.26 & 1.98 \\
\hline 0.63 & 31.07 & 51.48 & 20.83 & 37.34 & 7.40 & 3.73 \\
\hline
\end{tabular}

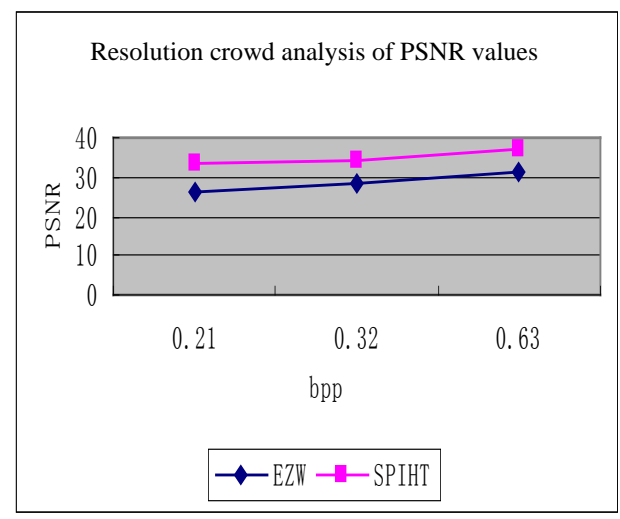

Fig.3 PSNR value analysis

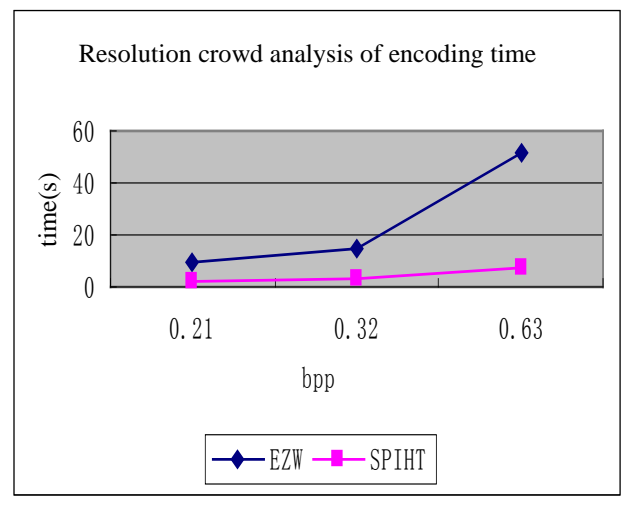

Fig.4 encoding time analysis

From Table 1 and Figure 3-5 it can be observed that PSNR of SPIHT is much higher than that of EZW, regardless of how large bpp(Bit Per Pixel) is. The improvement of PSNR( Peak Signal to Noise Ratio) over EZW is 7.48 at most and 6.57 in average. The encoding and decoding time is 
improved by about 7 times. from Figure 6 SPIHT algorithm is better than the visual effect of the reconstructed image EZW algorithm.

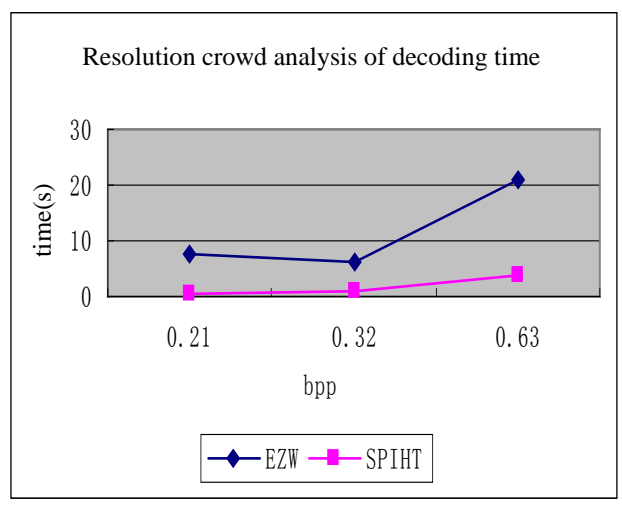

Fig.5 decoding time analysis

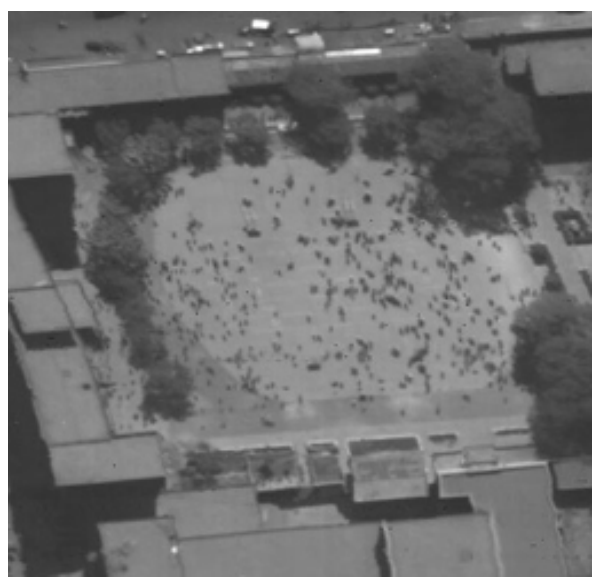

Fig. 6 Reconstructed images of resolution crowd a) Original image (resolution crowd)

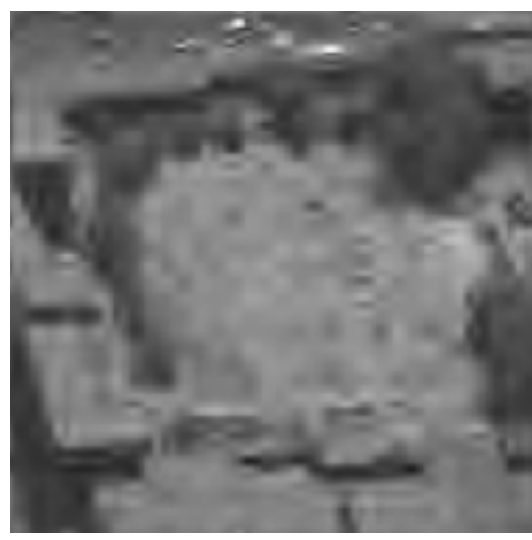

b)EZW bpp=0.21,PSNR=26.35

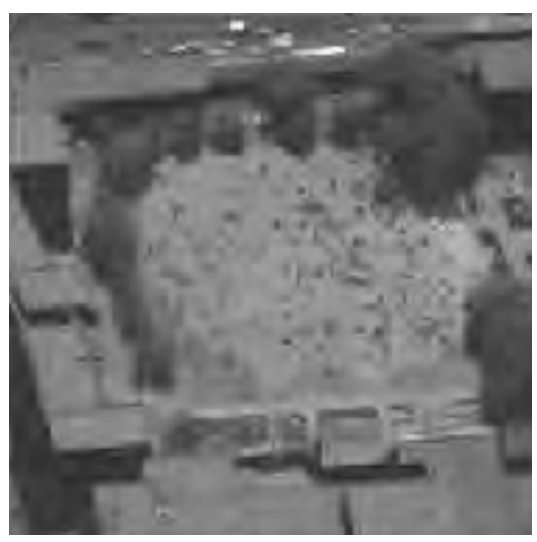

e)SPIHT bpp=0.21,PSNR=33.41

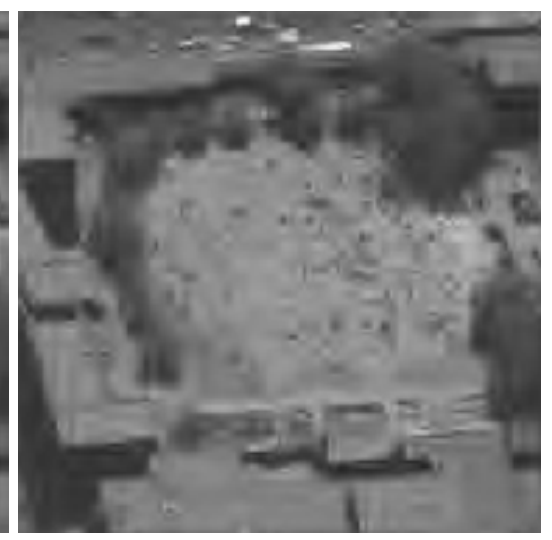

c) $\mathrm{EZW}$ bpp=0.32,PSNR=28.37

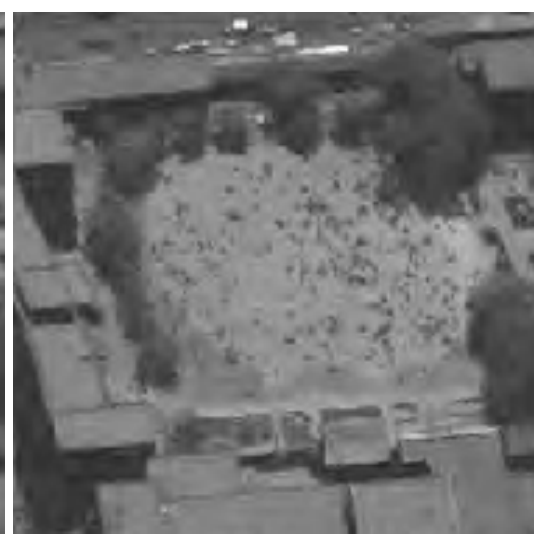

f) SPIHT bpp=0.32,PSNR=34.45

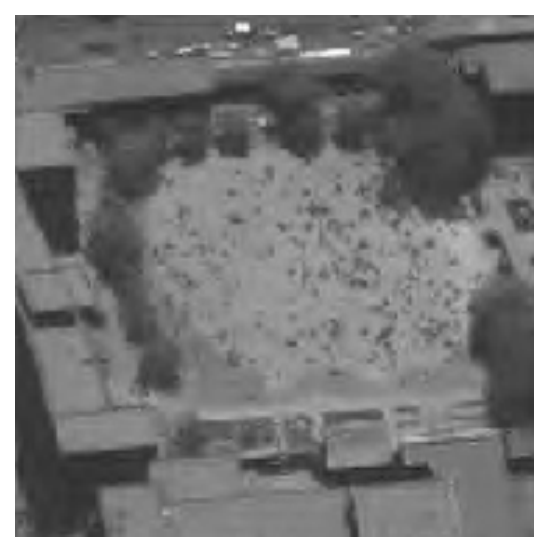

d) $\mathrm{EZW}$ bpp=0.63,PSNR=31.07

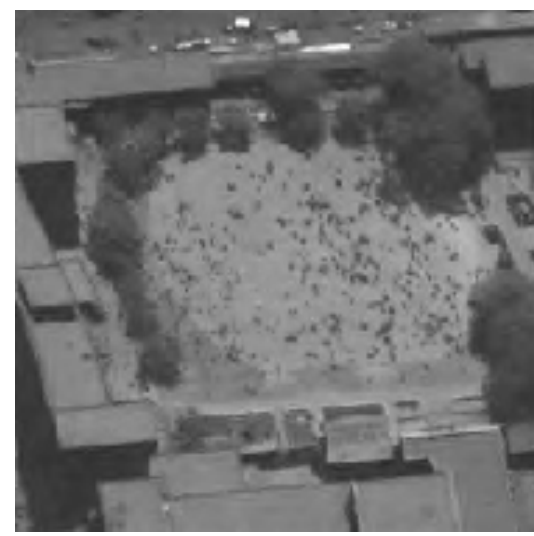

g) SPIHT bpp=0.63,PSNR=37.34

Fig. 6 Reconstructed images of resolution crowd

\section{Conclusion}

Wavelet transform is employed in this paper for three-level decomposition of remote sensing images. Study of the classic encoding algorithms shows that SPIHT provides higher-granularity set partitioning and can greatly improve compression efficiency compared with other classic algorithms. So it is a method that can compress wavelet zero tree images more efficiently than EZW does, resulting in higher compression ratio without entropy coding. In this context, SPIHT is adopted as the coding algorithm in this paper. Analysis and simulation of the algorithms are performed. 
Experimental results demonstrate that the PSNR values of wavelet transform coupled with SPIHT are much higher than that of the traditional wavelet compression algorithm. Moreover, both the compression and decompression time needed are reduced, and the human visual quality of reconstructed images is higher.

\section{Acknowledgements}

The work was financially supported by the surface project on science and technology research of the Education Department of Heilongjiang province (12521546).

\section{References}

[1] Yang Jian-guo,Wavelet Analysis and Its Engineering Application ,China Machine Press, PP. 6 33,2005.

[2] Rafael C. Gonzalez, Richard E.Woods,Ruan Qi-qi, Ruan Yu-zhi. Digital Image Processing,China Electronic Industry Press,PP. 327 334, 2003.

[3] KeLi,Research on Remote Sensing Image Real Time Compression Based on Wavelet Transform. PhD Dissertation from Chinese Academy of Science.

[4] J.M.Shapiro, Embedded Image Coding Using Zero-trees of Wavelet Coefficients,IEEE Trans. on Signal Processing,vol.12, pp. 3445 3462,1993.

[5] A.SaidW,A. Pearlman,A New Fast and Efficient Image Codec Based on Set Partitioning in Hierarchical Trees,EEE Trans on .Circuits and System for Video Technology,vol.6,pp. 243 248,1996.

[6] D. Taubman, E. Ordentlin,Embedded Block Coding in JPEG2000,Image Communication., vol.17,pp. 49 72,2002.

[7] A.SaidW, A.Pearlman,A New Fast and Efficient Image Codec Based on Set Partitioning in Hierarchical Trees,IEEE Trans. on Circuits and System For Video Technology,vol.6,pp. 243 248,1996.

[8] Hong Oh Kim, Rate Young Kim,Characterizations of biorthogonal wavelets which are associated with biorthogonal multiresolution analyses,Applied and computational Harmonic analysis,vol.11,pp. 263 272,2001. 